\title{
An Overview of Image-Guided Percutaneous Ablation of Renal Tumors
}

\author{
Majid Maybody, M.D. ${ }^{1}$
}

\section{ABSTRACT}

Although nephron-sparing surgery remains the gold standard treatment for small renal tumors, minimally invasive image-guided percutaneous ablation is becoming a viable alternative to operative resection. Percutaneous radiofrequency ablation (RFA) and cryoablation show high technical success rates, a relatively low incidence of residual or recurrent tumor, and competitive rates of patient survival. In this review, an overview of the current status of image-guided percutaneous ablation of renal tumors is presented, with a focus on procedure indications and patient selection, technical aspects of ablation procedures, and treatment outcomes and patient follow-up.

KEYWORDS: Percutaneous, ablation, radiofrequency, cryoablation, kidney, renal, tumor

\begin{abstract}
Objectives: Upon completion of this article, the reader should be able to explain the current status of image-guided percutaneous ablation of renal tumors, including procedure indications and patient selection, the technical aspects of ablation procedures, and treatment outcomes and patient follow-up.

Accreditation: Tufts University School of Medicine (TUSM) is accredited by the Accreditation Council for Continuing Medical Education to provide continuing medical education for physicians.

Credit: TUSM designates this educational activity for a maximum of 1 AMA PRA Category 1 Credit $^{\text {TM }}$. Physicians should only claim credit commensurate with the extent of their participation in the activity.
\end{abstract}

The incidence of renal tumors is rising in the United States, accounting for more than 50,000 new cases annually. ${ }^{1}$ This is mainly because of an increase in the diagnosis due to widespread use of cross-sectional imaging. ${ }^{2}$ Renal cell carcinoma constitutes $4 \%$ of all adult malignancies, ${ }^{3}$ and $\sim 70$ to $80 \%$ of patients present with localized low-stage disease. ${ }^{4,5}$ Nephron-sparing surgery continues to remain the gold standard treatment for small renal tumors. Although operative resection has been shown to be effective for treatment of small renal tumors and for preservation of renal function, it does have morbidity and mortality risks. ${ }^{6-8}$ Early laparoscopic renal cryoablation results have shown success and offer a less invasive technique for destroying small renal tumors. ${ }^{9}$ Image-guided percutaneous ablation of small renal tumors is less invasive, incurs less damage to uninvolved noncancerous renal tissue,${ }^{10}$ and is becoming a viable alternative to nephron-sparing surgery. ${ }^{10}$ The purpose of this article is to present an overview of imageguided percutaneous ablation of renal tumors.

\section{GENERAL CONCEPTS}

Radiofrequency ablation (RFA) and cryoablation are the two most commonly used ablation modalities. ${ }^{11,12}$ Each has unique technical features, and neither of the two has
${ }^{1}$ Section of Interventional Radiology, Department of Radiology, Memorial Sloan-Kettering Cancer Center, New York, New York.

Address for correspondence and reprint requests: Majid Maybody, M.D., Section of Interventional Radiology, Department of Radiology, Memorial Sloan-Kettering Cancer Center, 1275 York Avenue, H118A New York, NY 10065 (e-mail: maybodym@mskcc.org).
Image-Guided Tumor Ablation; Guest Editor, Ron C. Gaba, M.D. Semin Intervent Radiol 2010;27:261-267. Copyright (C) 2010 by Thieme Medical Publishers, Inc., 333 Seventh Avenue, New York, NY 10001, USA. Tel: +1(212) 584-4662.

DOI: http://dx.doi.org/10.1055/s-0030-1261784.

ISSN 0739-9529. 
Table 1 Comparison of Main Technical Features of Radiofrequency Ablation and Cryoablation in Percutaneous Image-Guided Ablation of Renal Tumors

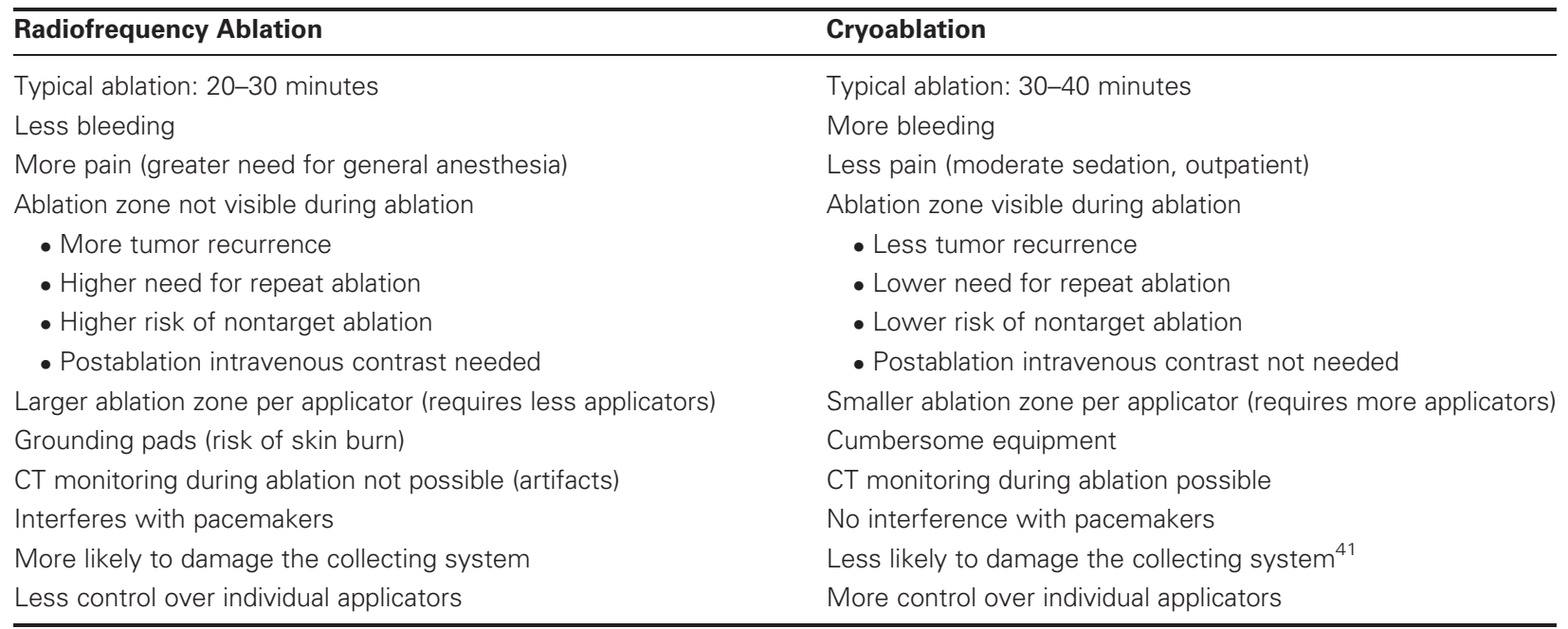

$\mathrm{CT}$, computed tomography.

clearly been proven to be superior to the other (Table 1). ${ }^{13}$ RFA destroys tumors by coagulation necrosis from temperatures above $60^{\circ} \mathrm{C}$, and cryoablation causes tumor necrosis by intracellular dehydration and disruption of cell membranes from temperatures below $20^{\circ} \mathrm{C}$. Both modalities are minimally invasive, as the thermal energy is emitted by placing one or more needlelike applicators into tumors. The ablation is performed under an imaging guidance modality such as ultrasound, computed tomography (CT), or magnetic resonance imaging (MRI). The procedures can be performed in an outpatient setting. The choice of percutaneous ablation modality is based on the availability of equipment, the interventional radiologist's experience with a partic-

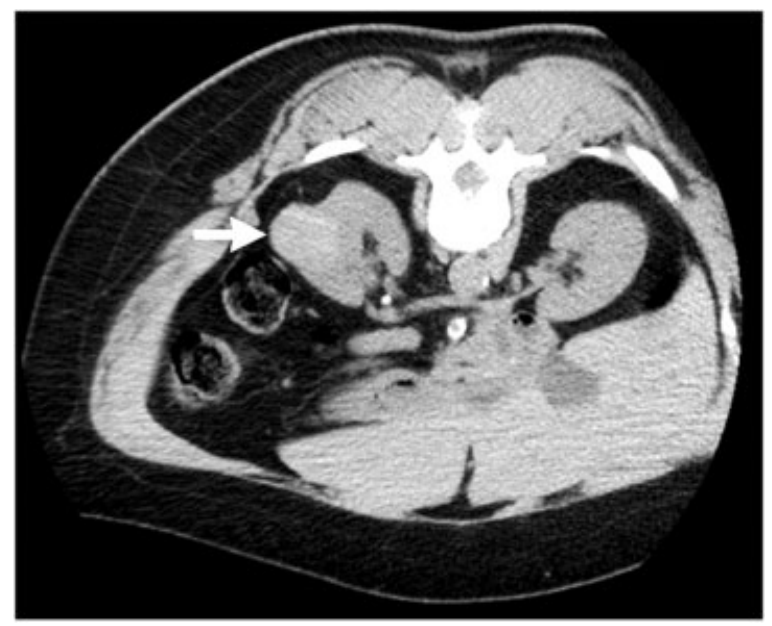

Figure 1 A 65-year-old woman with left-sided renal mass. Axial noncontrast computed tomography (CT) image performed prior to percutaneous ablation reveals 3.6-cm-diameter high attenuation exophytic mass (arrow) arising from posterior lateral aspect of left kidney. CT-guided biopsy confirmed papillary renal cell carcinoma. ular modality, and limitations based on anatomy and patient characteristics. The most important factors determining outcome of ablation are tumor size and location of tumor in the kidney. The best results are achieved in tumors $<4 \mathrm{~cm}$ in diameter. ${ }^{14,15}$ The least challenging tumor location is posterior exophytic (Fig. 1) and the most challenging location is central hilar. Central tumors carry a higher risk for bleeding, damage to hilar structures such as collecting system and vessels, and have a higher recurrence rate due to thermal heat-sink effect. Depending on the location of an anterior tumor, it may be approachable percutaneously. However, treatment of these tumors may require a transrenal or transhepatic approach. Tumors that abut central vessels have a higher risk of recurrence because of "thermal heatsink" phenomenon. This phenomenon occurs when the thermal energy required to achieve cytotoxicity during ablation is dissipated by the blood flow in the vessels abutting the tumor. All tumors less than $3 \mathrm{~cm}$ can be treated in one session and the need for more than one ablation session increases with tumor size $>3 \mathrm{~cm} .{ }^{14}$ Ablation of a 5 to $10 \mathrm{~mm}$ margin around the tumor is required to achieve the best results.

\section{PROCEDURE INDICATIONS AND PATIENT SELECTION}

Image-guided percutaneous ablations are especially ideal in patients who do not want to undergo surgery, elderly patients with significant medical comorbidities that preclude them from surgery, patients with renal insufficiency, solitary kidney, transplanted kidney, and multifocal tumors or patients with diseases such as von Hippel-Lindau, which predisposes them to develop multiple renal tumors. Documentation of renal cell carcinoma by needle biopsy is most often necessary, as 
up to $25 \%$ of renal masses $<3 \mathrm{~cm}$ are benign. Ideally, biopsy should be performed in a session separate from ablation. Tumors should be isolated to kidney with no evidence of vascular invasion or metastases. The best results are anticipated from tumors $<4 \mathrm{~cm}$ in diameter, although larger tumors have been successfully ablated percutaneously. The decision to perform percutaneous ablation is best made as a cooperative multidisciplinary agreement between an interventional radiologist and a urologist.

\section{PREPROCEDURE EVALUATION AND PRECAUTIONS}

Before the procedure, a physical exam should be performed, and the patient's medical history should be reviewed. Informed consent is required, and patients must understand that percutaneous ablations are relatively new procedures without proven long-term results. Allergies to contrast media, antibiotic medications, or anesthetic drugs should also be noted. Laboratory blood tests should include hematocrit, platelet count, prothrombin time, and international normalized ratio (INR), partial thromboplastin time (PTT), and creatinine with calculation of an estimated glomerular filtration rate (eGFR). A platelet count of more than 100,000 per mL, INR of less than 1.5, and normal PTT would ideally be met prior to the procedure.

Patients should not be acutely coagulopathic. Warfarin, aspirin, and clopidogrel should ideally be stopped at least 7 days before the procedure. The interventional radiologist should consult the referring physician prior to withholding anticoagulation medication. In patients with strict warfarin requirements, special arrangements can be made so ablation is performed using a "heparin window," in which warfarin is held and patients are systemically anticoagulated with heparin until the time of procedure.

Patients are instructed to abstain from eating for 6 hours prior to ablation so they are able to receive intravenous moderate sedation or anesthesia. They may take other routine oral medications with small sips of water. Modification of the insulin regimen in diabetics should be considered during the food restriction period prior to the procedure. Nephrology consultation may be needed prior to ablation in patients with chronic renal insufficiency (estimated GFR $<60 \mathrm{~mL} / \mathrm{min}$ ).

\section{IMAGING GUIDANCE}

Percutaneous image-guided ablation can be performed under ultrasound, computed tomography (CT), or magnetic resonance imaging (MRI) guidance. The tumor should be well visualized on the imaging modality planned for the procedure. The advantages of ultrasound are its "real-time" capability and lack of ionizing radia- tion. Ultrasound can be used for accurate placement of applicators before switching to CT or MRI for further monitoring. However, imaging with ultrasound is highly operator dependent and may be compromised in certain settings, such as patients with a large body habitus, the presence of abundant bowel gas, and when the tumor is near a focal loop of bowel that needs to be avoided. Another setback to ultrasound is the degradation of landmarks during ablation. In cryoablation, image degradation is caused by acoustic shadowing on the far side of the ice ball, and in RFA it is produced by microbubbles.

$\mathrm{CT}$ is not as operator dependent as ultrasound and is very accessible. Its wide field of view is excellent to cover the critical organs and structures that need to be avoided. CT scanning is much less sensitive to body habitus than is ultrasound and CT images are not affected by bowel gas. If $\mathrm{CT}$ is the chosen image guidance method, the target lesion should ideally be visible on a noncontrast examination. Percutaneous ablation can be performed using a conventional CT scanner or a CT scanner with real-time fluoroscopic capability.

MRI is the least commonly used imaging modality for percutaneous ablation. It provides excellent soft tissue resolution with multiplanar imaging capability and lacks ionizing radiation. MR fluoroscopic sequences can be used for real-time guidance. MR thermography can assess cytotoxic tissue temperatures noninvasively. Image-guided percutaneous ablation can be performed using a dedicated interventional magnet, a conventional solenoid magnet, or an open magnet. The ice ball is visualized as a zone of decreased signal intensity on T1and T2-weighted images. MRI-compatible ablation equipment is available to perform both RFA and cryoablation under MRI guidance. ${ }^{16,17}$

\section{ABLATION PROCEDURES}

The patient should be placed in the most comfortable position (prone, supine, lateral decubitus, or oblique) that also facilitates the procedure. Intravenous midazolam and fentanyl are the most commonly used medications for moderate sedation. The drug dosage is titrated for patient comfort and is monitored with telemetry and pulse oximetry by a sedation nurse or anesthesia team. At my institution, all percutaneous ablations are performed under general anesthesia with endotracheal intubation. If desired, a biopsy of the lesion can be performed in the same session prior to the ablation. A challenge with same day biopsy is that bleeding associated with the biopsy may obscure the tumor.

The procedure plan should be outlined by the interventional radiologist before the procedure. The number of applicators used for a particular tumor depends on the equipment used and the size of tumor (Fig. 2). Placement of more than one applicator is often 


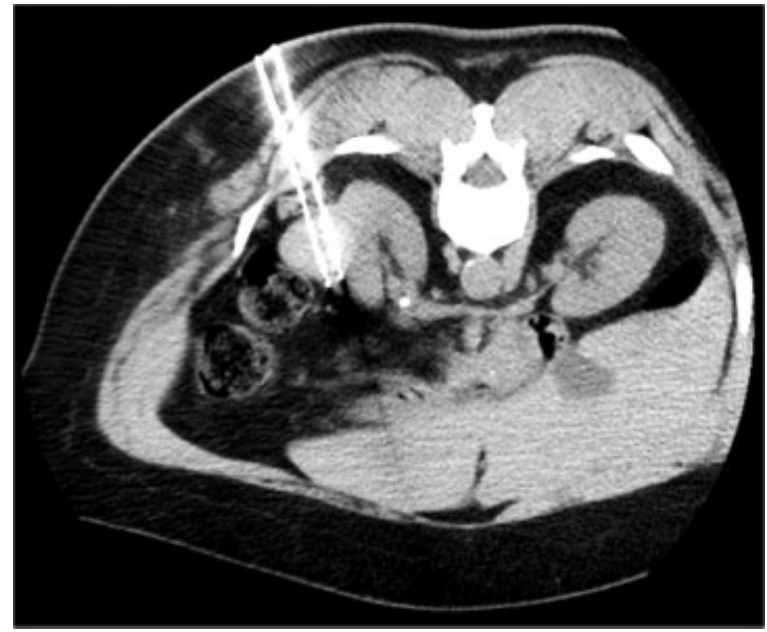

Figure 2 Percutaneous cryoablation of mass shown in Fig. 1. Intraprocedural computed tomography (CT) image shows appropriate position of two applicators within tumor, spaced appropriately to gain complete tumor coverage during ablation.

required to cover the entire tumor and the desired rim of surrounding parenchyma. The length of devices extending outside of the patient and their connecting cords, as well as patient positioning and the angle of applicator placement, all have major practical implications and should all be planned beforehand. Frequently, the injection of intravenous contrast medium during the procedure can help visualize relatively inconspicuous lesions. Smaller doses of iodinated contrast $(\sim 50 \mathrm{~mL})$ are usually sufficient for $\mathrm{CT}$ visualization and can allow for a repeat bolus later in the procedure if necessary. Similarly, there are circumstances when sonographic or MRI contrast agents may be useful.

Adjacent critical structures may be damaged when they are located within the target region. Occasionally, a second percutaneous needle can be inserted and carbon dioxide, water, air, or balloons may be placed to separate bowel, for instance, from the target tumor. ${ }^{15,18,19}$ It is important not to use saline for hydrodissection in RFA cases, as saline conducts current and may damage organs. Nonionic 5\% dextrose in water should be used instead. Certain patient positioning maneuvers or the interventional radiologist's hands may also be used to move away bowel loops from the tumor. Retrograde ureteral stents can be used to circulate warm or cold fluid during the ablation period to provide some protection to the ureter. ${ }^{15,20}$ The applicator can also be used as a lever to move the kidney away from adjacent structures. ${ }^{21}$ If a transpleural approach is inevitable, it can be pursued and any pneumothorax that develops may be treated accordingly. Alternatively, an iatrogenic pneumothorax or hydrothorax can be created to avoid potential damage to the lung.

Unless a tined RFA applicator is used, most applicators have no tines and produce an oval zone of ablation. After adequate positioning of the applicator(s) is complete, the tumor is ablated once or more according to the suggested manufacturer protocol. If the patient's renal function allows, a contrast-enhanced CT or MRI scan may be performed after removing all applicators. This will allow for an immediate assessment of the adequacy of the ablation. MRI-guided ablations can also be immediately assessed by MR thermography. Certain areas with suboptimal ablation can then be immediately re-treated during the same session. At a minimum, it is usually helpful to do a noncontrast scan of the treatment area following applicator removal to assess for complications.

An advantage of cryoablation over heat-based ablation modalities is its relative painlessness. ${ }^{22}$ This may be of importance for patients who cannot undergo general anesthesia or receive deep moderate sedation due to medical comorbidities.

In contrast to heat-based ablation modalities, the ice ball can be clearly seen with $\mathrm{CT}^{23,24}$ (Fig. 3), MRI, ${ }^{25}$ and ultrasound imaging. ${ }^{26}$ This imaging characteristic allows the operator to ascertain coverage of the tumor more confidently and also to better protect certain nearby critical structures. Because of this feature, cryoablated lesions tend to have less residual tumor. ${ }^{27}$ Intravenous contrast is usually not needed immediately following cryoablation to verify proper coverage of tumor by the ablation zone. This is a significant advantage for patients with renal insufficiency. The outer edge of the ice ball that is visualized is not cytotoxic.

Unlike the heat-based ablation modalities, freezing does not cauterize or coagulate vessels within the ablation zone. Hence, patients may be at slightly higher risk of bleeding during or immediately following

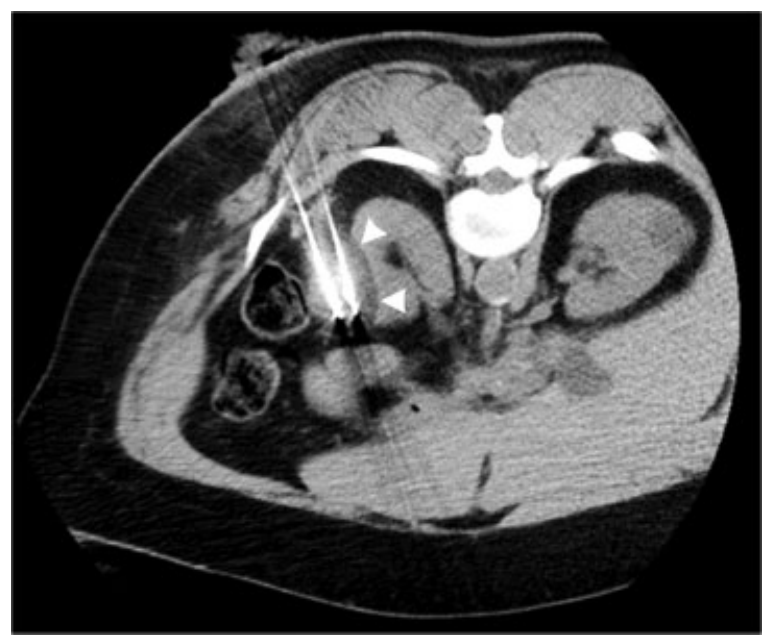

Figure 3 During cryoablation of mass shown in Figs. 1 and 2 , reduced attenuation ice ball (arrowheads) readily seen on noncontrast computed tomography (CT) after three 10-minute freeze cycles with interposed 5-minute thaw periods. At the discretion of the operator, no protective measures were taken to separate colon abutting lateral aspect of mass in this particular case. 


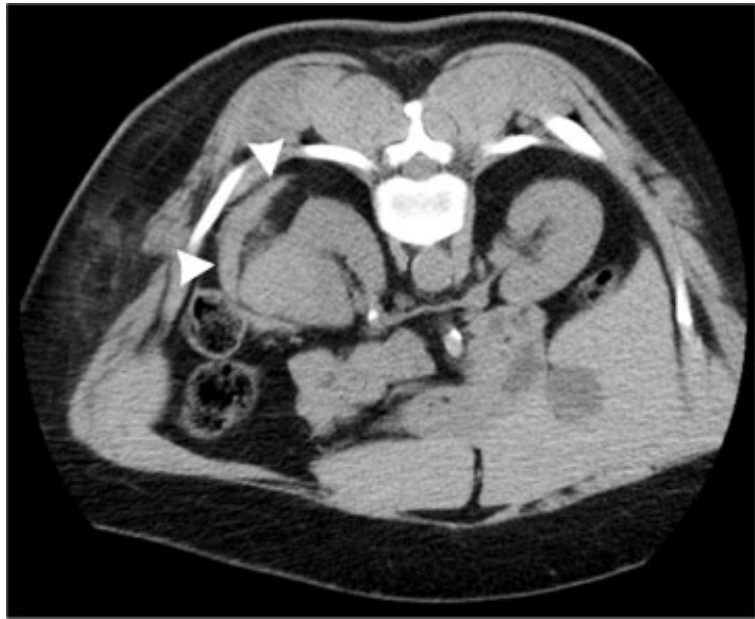

Figure 4 Final postablation computed tomography (CT) image following cryoablation of mass shown in Figs. 1 to 3 demonstrates small perinephric hematoma (arrowheads), which caused no clinical sequela.

cryoablation (Fig. 4). For this reason, patients with borderline coagulation status should have laboratory abnormalities more vigorously corrected. One relative limitation of current cryoablation applicators is the size of the ablation zone created by a single applicator as compared with the same size applicator from any of the heat-based ablation modalities. In general, each $2 \mathrm{~mm}$ applicator creates an $\sim 2 \mathrm{~cm}$ diameter of necrosis. ${ }^{28}$ For this reason, insertion of multiple simultaneous applicators is commonly needed to cover the entire tumor and desired margin of surrounding parenchyma. A simple rule-of-thumb is to position the applicators $1 \mathrm{~cm}$ from the margin of the tumor and 1 to $2 \mathrm{~cm}$ apart from each other, giving priority to the periphery of the tumor. ${ }^{23}$ It is important to remember that all the available cryoablation applicators currently have an extension cord attached to them. This cord contains tubing for gases, and in certain versions, temperature sensor wiring as well. The weight of the cord produces torque during and after placement of the applicator. When planning for cryoablation, it is important to make sure the trajectory of each applicator has enough purchase inside the patient so they are not constantly pulled or deviated by the torque produced by the cord. Depending on the type of the applicator used, a significant portion of the applicator and cord may extend outside of the patient. This may cause significant technical problems when performing cryoablation under CT or MRI guidance. When positioning the patient for the procedure, it is critical to remember to provide enough space between the patient and gantry so that the equipment is not contaminated during the procedure. Additionally, the interventional radiologist will have far more freedom to make fine adjustments during the procedure without having to take the patient out of the gantry repeatedly.

\section{TREATMENT OUTCOMES}

Technical success rates of 97 to $100 \%$ are reported with RFA and cryoablation. ${ }^{12,14,15}$ In a series of 616 patients, the incidence of residual or recurrent tumor was $13.4 \%$ with a single radiofrequency ablation and $3.9 \%$ with a single cryoablation. ${ }^{11}$ In this series, the overall incidence of residual or recurrent tumor after repeated ablation therapy in all patients was $4.2 \% .{ }^{11}$ The overall 2-year survival rate was $82.5 \%$, including patients who died of unrelated causes, and the metastasis-free survival rate was $97 \%$ at 2 years. ${ }^{11}$ Five-year local and distant tumorfree rate after RFA has been reported at $94 \% .{ }^{29}$ Similar success rates are reported with cryoablation, but the mean follow-up time period is shorter at 1 to 2 years. ${ }^{23}$

\section{OTHER ABLATION MODALITIES}

Microwave is a heat-based modality which involves deploying microwave energy $(300-3000 \mathrm{MHz})$ from a nontined applicator (antenna) into a tumor causing oscillation of ions, which creates heat resulting in coagulative necrosis. Advantages include faster ablation times and less susceptibility to thermal heat-sink effect. ${ }^{30}$

Laser is another heat-based ablation modality that deposits laser energy into the tumor via tiny fibers. Laser energy raises the tissue temperature and causes coagulative necrosis. Laser ablation has been successfully performed in few renal tumors, ${ }^{31}$ and can be done under CT or MRI guidance. Flexibility of the applicator outside of the patient is an advantage when working in a CT or MRI gantry. Relative small ablation zones mandate multiple applicator placement.

Irreversible electroporation (IRE) is a novel nonthermal ablation modality that causes apoptotic cell death by creating microscopic holes in cell membranes when cells are exposed to specific electrical fields. This modality is much faster than other minimally invasive ablation techniques. Another advantage of IRE is that it is not affected by thermal sink phenomenon. ${ }^{32}$ Preliminary animal and human experience is promising. The need for general anesthesia with paralytic agents is an issue, however.

High-intensity focused ultrasound (HIFU), is a noninvasive heat-based ablation modality. It causes coagulation necrosis by focusing a high-intensity ultrasonic beam onto a small volume of target lesion. Respiratory movements and overlying ribs are major problems with the use of HIFU in renal tumors. ${ }^{33,34}$

\section{COMPLICATIONS}

The rate of major complications from percutaneous ablation of renal tumors is $\sim 2$ to $6 \%{ }^{12,14,35}$ Complications include hemorrhage, pneumothorax, and bowel and nerve injury. In contrast to other heat-based ablation modalities, cryoablation does not cauterize blood vessels 
within the ablation zone. Therefore, hemorrhage at the ablation zone after removal of applicators appears to be slightly more common when compared with heat-based ablation modalities. Hemorrhage after cryoablation is most often self-limited and is managed with conservative management. Damage to the renal collecting system and the neighboring structures such as nerves and bowel are among other reported complications.

\section{PATIENT FOLLOW-UP}

If the patient's renal function allows, the ablated tumor should be evaluated by CT or MRI without and with intravenous contrast at 1, 3, 6, 12, 18, and 24 months after ablation. Early postablation studies show the ablation zone as a nonenhancing area surrounded by a thin smooth rim enhancement. ${ }^{36}$ This rim is considered a physiologic response to thermal ablation and disappears within 3 months. ${ }^{37}$ Ideally, the ablation zone should include the entire tumor and the expected 5 to $10 \mathrm{~mm}$ noncancerous rim. The ablation zone appears T1 hyperintense and T2 hypointense. ${ }^{38,39}$ The ablation zone continues to decrease in size over time. ${ }^{37}$ Complete disappearance of cryoablated tumors over time has been reported. ${ }^{40} \mathrm{~A}$ thin curvilinear hyperattenuating rim or halo on $\mathrm{CT}$ imaging, which is hypointense on T1-weighted images, is commonly seen parallel to the tumor extending to the perinephric fat. This halo may persist for several months after treatment. ${ }^{38,39}$ Nodular or irregular enhancement within the ablation zone and enlargement of ablation zone is considered suspicious for residual tumor or recurrence. ${ }^{39}$ These areas may be biopsied and retreated by another session of ablation.

\section{CONCLUSION}

Nephron-sparing surgery continues to remain the gold standard treatment for small renal tumors. Imageguided percutaneous ablation is a viable option for patients who cannot undergo surgery because of medical comorbidities. Early clinical experience with these ablation modalities is promising.

\section{REFERENCES}

1. Chow WH, Devesa SS, Warren JL, Fraumeni JF Jr. Rising incidence of renal cell cancer in the United States. JAMA 1999;281:1628-1631

2. Pantuck AJ, Zisman A, Belldegrun AS. The changing natural history of renal cell carcinoma. J Urol 2001;166:1611-1623

3. American Cancer Society. Cancer Statistics 2008. Atlanta, GA: American Cancer Society; 2008

4. Luciani LG, Cestari R, Tallarigo C. Incidental renal cell carcinoma-age and stage characterization and clinical implications: study of 1092 patients (1982-1997). Urology 2000;56: $58-62$
5. Janzen NK, Kim HL, Figlin RA, et al. Surveillance after radical or nephron sparing surgery for localized renal cell carcinoma and management of recurrent disease. Urol Clin North Am 2003;30:843-852

6. Fergany AF, Hafez KS, Novick AC. Long-term results of nephron sparing surgery for localized renal cell carcinoma: 10-year follow up. J Urol 2000;163:442-445

7. Gill IS, Kavoussi LR, Lane BR, et al. Comparison of 1,800 laparoscopic and open partial nephrectomies for single renal tumors. J Urol 2007;178:41-46

8. Breda A, Finelli A, Janetschek G, Porpiglia F, Montorsi F. Complications of laparoscopic surgery for renal masses: prevention, management, and comparison with the open experience. Eur Urol 2009;55:836-850

9. Spaliviero M, Moinzadeh A, Gill IS. Laparoscopic cryotherapy for renal tumors. Technol Cancer Res Treat 2004; 3:177-180

10. Hui GC, Tuncali K, Tatli S, Morrison PR, Silverman SG. Comparison of percutaneous and surgical approaches to renal tumor ablation: metaanalysis of effectiveness and complication rates. J Vasc Interv Radiol 2008;19:13111320

11. Gervais DA, McGovern FJ, Wood BJ, et al. Radiofrequency ablation of renal cell carcinoma: early clinical experience. Radiology 2000;217:665-672

12. Atwell TD, Farrell MA, Leibovich BC, et al. Percutaneous renal cryoablation: experience treating 115 tumors. J Urol 2008;179:2136-2141

13. Maybody M, Solomon SB. Image-guided percutaneous cryoablation of renal tumors. Tech Vasc Interv Radiol 2007;10:140-148

14. Boss A, Clasen S, Kuczyk M, et al. Magnetic resonanceguided percutaneous radiofrequency ablation of renal cell carcinomas: a pilot clinical study. Invest Radiol 2005;40: 583-590

15. Miki K, Shimomura T, Yamada H, et al. Percutaneous cryoablation of renal cell carcinoma guided by horizontal open magnetic resonance imaging. Int J Urol 2006;13: 880-884

16. Farrell MA, Charboneau JW, Callstrom MR, et al. Paranephric water instillation: a technique to prevent bowel injury during percutaneous renal radiofrequency ablation. AJR Am J Roentgenol 2003;181:1315-1317

17. Kam AW, Littrup PJ, Walther MM, et al. Thermal protection during percutaneous thermal ablation of renal cell carcinoma. J Vasc Interv Radiol 2004;15:753-758

18. Gervais DA, Arellano RS, McGovern FJ, McDougal WS, Mueller PR. Radiofrequency ablation of renal cell carcinoma: part 2, lessons learned with ablation of 100 tumors. AJR Am J Roentgenol 2005;185:72-80

19. Cantwell CP, Wah TM, Gervais DA, et al. Protecting the ureter during radiofrequency ablation of renal cell cancer: a pilot study of retrograde pyeloperfusion with cooled dextrose 5\% in water. J Vasc Interv Radiol 2008;19:10341040

20. Park BK, Kim CK. Using an electrode as a lever to increase the distance between renal cell carcinoma and bowel during CT-guided radiofrequency ablation. Eur Radiol 2008;18: 743-746

21. Allaf ME, Varkarakis IM, Bhayani SB, et al. Pain control requirements for percutaneous ablation of renal tumors: cryoablation versus radiofrequency ablation - initial observations. Radiology 2005;237:366-370 
22. Littrup PJ, Ahmed A, Aoun HD, et al. CT-guided percutaneous cryotherapy of renal masses. J Vasc Interv Radiol 2007;18:383-392

23. Solomon SB, Chan DY, Jarrett TW. Percutaneous cryotherapy of kidney tumors. Am J Urol Rev 2004;2:369-371

24. Silverman SG, Tuncali K, van Sonnenberg E, et al. Renal tumors MR imaging-guided percutaneous cryotherapy initial experience in 23 patients. Radiology 2005;236: 716-724

25. Gill IS, Novick AC, Meraney AM, et al. Laparoscopic renal cryoablation in 32 patients. Urology 2000;56:748-753

26. Matin SF, Ahrar K, Cadeddu JA, et al. Residual and recurrent disease following renal energy ablative therapy: a multi-institutional study. J Urol 2006;176:1973-1977

27. Permpongkosol S, Nicol TL, Link RE, et al. Differences in ablation size in porcine kidney, liver, and lung after cryoablation using the same ablation protocol. AJR Am J Roentgenol 2007;188:1028-1032

28. Gervais DA, McGovern FJ, Arellano RS, McDougal WS, Mueller PR. Radiofrequency ablation of renal cell carcinoma. Part 1. Indications, results, and role in patient management over a 6-year period and ablation of 100 tumors. AJR Am J Roentgenol 2005;185:64-71

29. McDougal WS, Gervais DA, McGovern FJ, Mueller PR. Long-term follow-up of patients with renal cell carcinoma treated with radio frequency ablation with curative intent. J Urol 2005;174:61-63

30. Simon CJ, Dupuy DE, Mayo-Smith WW. Microwave ablation: principles and applications. Radiographics 2005;25: S69-S83

31. Dick EA, Joarder R, De Jode MG, Wragg P, Vale JA, Gedroyc WM. Magnetic resonance imaging-guided laser thermal ablation of renal tumours. BJU Int 2002;90(9):814-822
32. Rubinsky B, Onik G, Mikus P. Irreversible electroporation: a new ablation modality: clinical implications. Technol Cancer Res Treat 2007;6:37-48

33. Marberger M, Schatzl G, Kranston D, Kennedy JE. Extracorporeal ablation of renal tumors with high intensity focused ultrasound. BJU Int 2005;95:52-55

34. Klatte T, Marberger M. High-intensity focused ultrasound for the treatment of renal masses: current status and future potential. Curr Opin Urol 2009;19:188-191

35. Johnson DB, Solomon SB, Su LM, et al. Defining the complications of cryoablation and radiofrequency ablation of small renal tumors: a multi-institutional review. J Urol 2004; 172:874-877

36. Smith S, Gillams A. Imaging appearances following thermal ablation. Clin Radiol 2008;63:1-11

37. Merkle EM, Nour SG, Lewin JS. MR imaging follow-up after percutaneous radiofrequency ablation of renal cell carcinoma: findings in 18 patients during first 6 months. Radiology 2005;235:1065-1071

38. Uppot RN, Silverman SG, Zagoria RJ, Tuncali K, Childs DD, Gervais DA. Imaging-guided percutaneous ablation of renal cell carcinoma: a primer of how we do it. AJR Am J Roentgenol 2009;192:1558-1570

39. Kawamoto S, Solomon SB, Bluemke DA, Fishman EK. Computed tomography and magnetic resonance imaging appearance of renal neoplasms after radiofrequency ablation and cryoablation. Semin Ultrasound CT MR 2009;30:67-77

40. Gill IS, Remer EM, Hasan WA, et al. Renal cryoablation: outcome at 3 years. J Urol 2005;173:1903-1907

41. Janzen NK, Perry KT, Han KR, et al. The effects of intentional cryoablation and radiofrequency ablation of renal tissue involving the collecting system in a porcine model. J Urol 2005;173:1368-1374 